DOI: $10.1515 / \mathrm{rrlm}-2016-0042$

\title{
Complete blood count and differential in diagnosis of early onset neonatal sepsis
}

\section{Parametrii hemogramei complete și a formulei leucocitare în diagnosticul sepsisului neonatal cu debut precoce}

\author{
Maria Livia Ognean ${ }^{1}$, Adrian Boicean ${ }^{2}$, Floredana-Laura Șular ${ }^{3, *}$, Manuela Cucerea ${ }^{3}$ \\ ${ }^{l}$ Clinical County Emergency Hospital Sibiu, Romania, ${ }^{2}$ Faculty of Medicine, University Lucian Blaga, \\ Sibiu, Romania, ${ }^{3}$ University of Medicine and Pharmacy Tîrgu Mureș, Romania
}

Received: $2^{\text {th }}$ June 2016; Accepted: 14 ${ }^{\text {th }}$ November 2016; Published: $21^{\text {th }}$ November 2016.

\section{Neonatal sepsis - incidence, morbidity and mortality}

Neonatal sepsis, one of the main causes of neonatal morbidity and mortality (1), is a complex clinical syndrome occurring in 1-8.6/1000 live births (2) as a result of the systemic inflammatory response to infection during the neonatal period (3). Early onset sepsis (EOS) is defined as sepsis occurring during the first 72 hours of life as a fetal response to an ascending infection (from the birth canal) or to the hematogenous dissemination of a maternal infection (4).

Sepsis is a complex condition initiated by a pathogen and mediated by cytokines followed by immune, inflammatory, and coagulation homeostasis disturbances, its evolution being dictated by a complicated balance between pro inflammatory and anti inflammatory factors (5) Most of the short and long-term complications of the neonatal sepsis are strictly related to inflammatory mediators. Neonatal sepsis is associated with a mortality rate that ranges from 13 to $60 \%$ $(6,7)$ in spite of improved antibiotic therapy and care and an increased morbidity in survivors.

"Suspected sepsis" is one of the most frequently encountered diagnosis in neonatology because: a) a large number of newborns are evaluated for early or late sepsis based on risk factors and for fear of missing a correct diagnosis and a prompt treatment $(8)$; b) in neonates, the clinical signs of infection are not specific, late, and the differential diagnosis with neonatal respiratory distress syndrome, aspiration syndromes, or neonatal maladaptation to extrauterine life is difficult (8-10); c) blood culture - the golden standard in neonatal sepsis diagnosis - provides late information, has a poor accuracy, and is not universally available (11); and d) we do not have yet an ideal diagnostic tool for neonatal infection (12). Therefore, diagnosis of neonatal sepsis is still a challenge for neonatal medicine. Antibiotic therapy is often initiated based upon clinical suspicion and/or the presence of risk

* Corresponding author: Floredana-Laura Şular, University of Medicine and Pharmacy Tîrgu Mureș, Gheorghe Marinescu 38, Tîrgu Mureș, Romania e-mail: floredana.sular@gmail.com 
factors, leading to excessive antibiotic therapy. Very often the diagnosis criteria for neonatal sepsis consists in documenting an infection in a newborn with severe systemic disease in which all possible noninfectious explanations for the patient's altered physiological status are either ruled out or unlikely (13).

\section{Blood culture - the "gold standard" test}

The "gold standard", definitive test for neonatal sepsis is the isolation of the pathogen from blood (9). However, its accuracy is influenced by multiple factors a) contamination during sampling; b) sampling after antibiotic therapy was started; c) insufficient sampling volume; d) low colony count bacteriemia (14). Accuracy of the blood culture varies between 8 and $73 \%$ in various studies $(6,11,15)$.

\section{Ideal markers for neonatal sepsis}

As seen by experts, the ideal marker for neonatal sepsis must fulfill the following characteristics:

- Clinically, it must have well defined reference values that allow interlaboratory comparison, sensibility towards $100 \%$, specificity over $85 \%$, positive predictive value over $85 \%$, negative predictive value over $100 \%$, power to detect infection in early stages, ability to differentiate between different types of pathologic agents (viruses versus bacteria, for example), and allow guidance and monitoring of the antibiotic therapy $(6,9,10)$;

- In the laboratory, the ideal marker must be a stable product, with a time window adequate for sampling (sustained increase and decrease of its values within 48 hours after the onset of the clinical manifestations), must be quantitative, allow use of small sample volumes, the measurement method must be simple, rapid, with comparable results between laboratories, and with a low cost (16).

\section{Current consensus of neonatal sepsis lab- oratory diagnosis}

Despite hundreds of published studies, there is still no consensus regarding the best screening test or panel of tests for rapid detection of neonatal sepsis. Recently, new acute phase proteins, cytokines, cell surface antigens, and bacterial genome are used to improve the neonatal sepsis diagnosis but data are still under evaluation and most of these tests are either not clinically available or they are expensive $(9,16)$.

\section{Complete blood count and differential - reference values and influencing factors}

Evaluation of complete blood count (CBC) and differential was the first test used to diagnose neonatal sepsis, hematological indices still being the most extensively used in practice, currently in association with new markers for infection $(11,12,17,18)$. The different rate of neutrophil release from bone marrow into circulation, the egress rate of the neutrophils from blood into the tissues, and their distribution in circulation and tissues are responsible for the morphological and concentration abnormalities of the circulating neutrophils seen in neonatal sepsis (17).

Since 1979, reference values for blood neutrophil concentration in late preterm and term infants during the first 700 hours of life defined by Manroe et al. were extensively used for EOS evaluation. Mouzinho et al. (19) defined the reference values, upper and lower limits for blood neutrophil concentration in very low birth weight (VLBW) preterm infants. The brutal transition from the protective intrauterine environment to extrauterine life is characterized by extreme physiological changes required for survival. Some of the metabolic and physiological processes continue to undergo changes during the first days of life and these may influence sometimes dramatically the results offered by 
laboratory tests. These influences must be considered when interpreting the values offered by laboratory tests (9). Therefore, beyond individual variability, a certain value of a laboratory test must not be considered as static. Schmutz et al. (18) showed that the neutrophil count increases in the first hours after birth and peaks at 6-8 hours for infants $\geq 28$ weeks gestation, and later, at 24 hours, in those $<28$ weeks gestation. Immature/total neutrophil (I:T) ratio, one of the most valuable hematological indices, is $\leq 0.16$ at birth and decreases to 0.12 at 72 hours of life in healthy neonates (20). After birth, the absolute immature neutrophil count follows the same pattern as the absolute neutrophil count (ANC), registering a peak value at approximately 12 hours of life (20). Labor and catecholamine release, as part of the adaptation process to extrauterine life, may be responsible for the transient increase of leukocyte and neutrophil count in the first day of life $(18,21)$. In most of the EOS cases the leukocyte count, the leukocyte characteristics, and indices become abnormal around 12 hours of life $(10,22)$.

The peripheral smear is useful for EOS diagnosis as it identifies characteristic morphologic changes of the neutrophils and monocytes: abnormal density of the nuclear chromatin, toxic granulations, vacuolization, Döhle bodies, left shift (23). However, the method is time consuming, requires manual examination and experience, the results are biased by subjective interpretation, reduced reproducibility, and it is based on the analysis of just hundred of cells for any given sample $(17,23)$. The computerized analysis provides information from thousands of leukocytes, measures the size of the cells, evaluates the internal cellular composition, granularity, and complexity of the nuclear shape (23) and is less liable to subjective interpretative errors (24). Yet, errors and biases are still possible: when using the impedance principle, the leukocyte count may be affected by the osmotic resistance of some neonatal red blood cells and/or by the high neonatal normoblast count resulting in a falsely increased number of white blood cells; incorrect blood : anticoagulant ratio in small tubes may also give rise to errors $(23,24)$.

Interpretation of the $\mathrm{CBC}$ parameters as normal or abnormal and use of different classification systems makes the interpretation of the borderline or grossly abnormal values difficult (22).

\section{Complete blood count and differential - utility in screening for early onset sepsis}

Total leukocyte count (TLC), total neutrophil count, ANC, immature neutrophil count, immature/total neutrophil ratio (I:T ratio), immature/ mature neutrophil ratio (I:M ratio), neutrophil degenerative changes (vacuolization, toxic granulations, and Döhle bodies), and platelet count are the most used hematological parameters for EOS evaluation $(7,9)$. Most of the hematological screening panels for EOS use a total leukocyte count $<5000$ cells $/ \mathrm{mm}^{3}$ or $>20.000$ cells $/ \mathrm{mm}^{3}$, an I:T ratio $>0.2$, and total neutrophil counts outside the normal range $(7,8)$.

The vast majority of studies in the literature conclude that hematological indices have a low sensibility and specificity in identifying EOS, being more useful in identifying newborns with low risk for sepsis. Six to 12 hours are necessary before the effect of the inflammatory response on the number and ratio of immature and mature neutrophil occurs $(9,17,22)$. An extensive review performed by da Silva et al. (25) that found a large range of sensibilities - 17-90\% - and specificities - 31-100\% - for leukocytes and leukocyte indices in EOS screening, explained the results by laboratory experience variances, different postnatal age of the patients, and influence of noninfectious factors affecting neutrophils parameters and concluded that serial measurements of the leukocyte indices may increase the accuracy of EOS diagnosis and allow monitor- 
ing of the treatment. Sonawane et al. (26) found that CBC has a sensibility of 37.5 , specificity of $75 \%$, positive predictive value (PPV) of $69.2 \%$ and negative predictive value (NPV) of $44.4 \%$ for neonatal sepsis detection and underlined that CBC has a higher specificity compared to CRP. In EOS detection, Schmutz et al. (18) identified a sensibility $>90 \%$ for abnormal immature neutrophil count, I:T ratio, and ANC, and a specificity $>90 \%$ for abnormal I:M ratio and I:T ratio, associated with neutrophil degenerative changes and decreased platelet count, and a PPV of 94.4\% for abnormal I:M ratio. A more recent review, performed by Bhat and Rao (6), based on 17 studies, showed that a leukocyte count $<5000$ cells $/ \mathrm{mm}^{3}$ or $>20.000$ cells $/ \mathrm{mm}^{3}$, a platelet count $<150.000 / \mathrm{mm}^{3}$, an I:T ratio $\geq$ 0.2 , and the presence of vacuoles in the cytoplasm or toxic granulation on the peripheral smear suggest EOS with sensibilities that range between 15.6 and $81 \%$, but none of the studied parameters predicted bacteriemia in a satisfactory manner. Murphy and Weiner (21) demonstrated that two CBCs, performed at 8-12 hours interval, and a negative blood culture accurately exclude neonatal sepsis (up to $100 \%$ NPV), but the low sensitivity and PPV are not useful to guide therapy.

Leukopenia was found to be a better predictor for neonatal sepsis compared to leukocitosis (sensitivity of $87.5 \%$ versus $25 \%$ ) and is more useful in infections with gram negative germs (27). Others have demonstrated that TLC increases in severe neonatal infections (both mature and immature cells) possible secondary to growth factors and cytokine release that stimulate the bone marrow production (28). Typically, neonates with viral infections have a normal TLC or a slightly reduced leukopenia (8).

Neutropenia is considered a better and more specific marker for EOS compared to neutrophilia because less factors - other than infection
- cause a decrease in neutrophil count $(17,18)$.

The release of neutrophils from the bone marrow in response to infection increases the number of immature neutrophils in circulation and shifts the differential count to the left. da Silva et al. (25) found that an I:T ratio $>0.2$ has a sensibility of $90 \%$ and NPV of $98 \%$ for EOS detection since the ratio is less influenced by non infectious factors compared to other neutrophil indices, in accordance with the results of other studies $(10,17,25)$. The combination of two normal values of the I:T ratio and a negative blood culture at 24 hours accurately allows EOS exclusion (21). On the contrary, some investigators found increased I:T ratios in $25-50 \%$ of the newborns without infection (17) and Sonawane et al. (26) found that I:T ratio has a sensibility of only $62.5 \%$ and NPV of $50 \%$ for sepsis detection. The $\mathrm{I}: \mathrm{M}$ ratio is also increased in EOS and, similar to $\mathrm{I}: T$ ratio, it is considered more helpful for EOS diagnosis compared to TLC (12). Both ANC and $\mathrm{I}: \mathrm{T}$ ratio are better at excluding infection than at identifying newborns with sepsis (18).

The morphological changes of neutrophils - toxic granulations and/or vacuolization - on the peripheral smear are, most often, late signs of EOS and indicate a severe infection but these changes never occur in healthy neonates (17).

Automatic analysis using VCS technology (V - volume, C - conductivity, S - scatter) allows detection of morphological changes of the immature and reactive neutrophils. Also neutrophils mean volume (MNV) and volume distribution width (VDW) can be determined and Celik et al. (23) have found that these two parameters can be helpful for EOS detection.

Thrombocytopenia, due to increased destruction, sequestration secondary to infection, failed platelet production secondary to reduced megakaryocytes, or damaging effect of endotoxins $(11,12)$ is frequently associated with neonatal sepsis $(11,29)$ but is, usually, a late sign of 
infection, indicating a poor prognosis (18) and cannot be used to guide antibiotic therapy since thrombocytopenia may persist even weeks after an infectious episode $(17,30)$.

Some new hematological markers of infection are tested. The granulocyte colony stimulating factor produced by the bone marrow facilitates neutrophil proliferation and differentiation, and was demonstrated to be a relatively early marker of EOS and bacterial infection (31).

\section{Complete blood count parameters used in combination with other markers for in- fection}

Most leukocyte parameters have a weak predictive accuracy for EOS diagnosis especially if considered separately while different combinations of these parameters may reach $90 \%$ sensibility (6). Sankar et al. (20) had demonstrated that 2 or more than 2 abnormal neutrophil parameters can detect EOS with $93-100 \%$ sensibility, $83 \%$ specificity, $27 \% \mathrm{PPV}$, and $100 \% \mathrm{NPV}$, suggesting that if the hematological screening identifies 2 or more than 2 abnormal neutrophil parameters, antibiotic therapy should be started. An abnormally reduced TLC, neutropenia, and an increased I:T ratio were associated with increased risk of infection - odds ratio over 5.38 - even though their sensitivity was low - $<54.5 \%$ - in a large cohort study of 166.092 neonates suspected of EOS (10), results that had been confirmed by another large cross-sectional study performed by Newman et al. (22).

Since sensitivity and specificity of the hematological indices are increasing when more parameters are evaluated, hematological scoring systems (HSS) were proposed for neonatal sepsis detection. Such scores are available, relatively rapid, with low cost, and practically accessible in most laboratories but they still need simplification and standardization $(12,18)$. Makkar et al. (11) found that the combination between in- creased I:T ratio and immature neutrophil count had the best sensitivity for EOS and probable EOS prediction, while increased I:T and I:M ratios were the most specific; a higher score was associated with a higher accuracy and probability of EOS, allowing a satisfactory identification of EOS and decision to treat.

Another, more complicated, scoring system for EOS detection was proposed by Selimovic et al. (15) who included CBC parameters - TLC, I:T ratio, I:M ratio - and CRP and, in a group of 341 newborns, this score had a sensitivity of $73 \%$ and NPV of $75 \%$. Usha et al (32) recently published another complex hematological score, stratified for EOS and late onset sepsis (LOS) diagnosis, and found low accuracy for EOS diagnosis at scores of 3-4.

$\mathrm{C}$-reactive protein is an acute phase reactant, a protein synthesized by the liver, whose values are increasing in inflammations due to infection or tissue damage, with a sensitivity of $47-100 \%$ for detection of bacterial sepsis $(16,32)$. Most of the recently used screening panels for EOS detection include $\mathrm{CBC}$ and $\mathrm{CRP}$, more rarely procalcitonin or, even more rarely, cytokines, thus increasing slightly the sensitivity and more significantly the NPV compared to the individual accuracy of the included markers (9). As in the case of $\mathrm{CBC}$, the CRP increase as response to inflammation needs a few hours after the infectious contact, therefore repeated CRP and CBC are followed by increased sensitivity and specificity. Makkar et al. (11) showed that decreased TNL $(<10$ X 109/L) and total neutrophil count $(<8$ X109/L), and increased I:T ratio $(>0.16)$ and $\mathrm{I}: \mathrm{M}$ ratio $(>0.25)$, and $\mathrm{CRP}(>0.6 \mathrm{mg} / \mathrm{dL})$ are useful tests for EOS diagnosis in the first 3 days of life, detecting $90 \%$ of the sepsis cases proven by blood culture. Combined screening panels can detect apparently healthy neonates "at risk" who may not need treatment or in which the treatment may be stopped (17). 


\section{Current attitude. Final comments}

Diagnosis of neonatal sepsis is still a challenge for neonatal medicine while any delay of the treatment increases the morbidity and mortality. There is still no consensus regarding the best screening test or panel of tests for EOS rapid diagnosis (9). Furthermore, very few reliable conclusions can be drawn from the published data since most of the studies are based on a limited number of patients who, in many of the studies, are not stratified on gestational age and/or postnatal age, the methodology is not sufficiently rigorous as regards the accuracy of the laboratory tests, and selection of the cut-off values of the tests is extremely heterogeneous.

For many years now, $\mathrm{CBC}$, with its limits and biases, continues to be the most frequently used and useful screening test for neonatal EOS (11), only recently CRP being added in order to increase the predictive accuracy (9). Tests with better predictive accuracy - such as procalcitonin, cytokines, etc. - are not universally available and are expensive. Still, none of the actual markers of infection is sufficiently sensitive or specific to definitely influence the decision to start antibiotic therapy independent of the clinical signs, most of the tests used to diagnose infection being more useful for identification of the neonates without infection (17). Therefore we, the clinicians, are obliged to have a pragmatic approach as regards the use of available tests for neonatal EOS diagnosis and antibiotic therapy guidance. Interpretation of the screening tests used for early diagnosis of EOS must be done in accordance with the risk factors and clinical signs and symptoms suggesting infection. Presence of the clinical signs or symptoms suggesting infection should prompt antibiotic therapy even if the screening tests are negative (22). Hopefully, a better understanding of the inflammatory response in preterm and term neonates may lead to improved screening for neonatal sepsis.

\section{References}

40. Camacho-Gonzalez A, Spearman PW, Stoll BJ. Neonatal infectious diseases: evaluation of neonatal sepsis. Pediatr Clin North Am. 2013 Apr;60:367-89. DOI: $10.1016 /$ j.pcl.2012.12.003.

41. Isaac D, Barfield C, Clothier T, Darlow B. Late onset infections of infants in neonatal units. J Paediatr Child Health. 1996;32:158-61. DOI: 10.1111/j.14401754.1996.tb00914.x.

42. Pal K, Samanta AK, Singh RA. A comparative study of early onset sepsis versus late onset neonatal sepsis with special reference to bacteriological, demographic and clinical profile. Int J Cur Res Rev. 2014 June;6(03):7-15.

43. Haque KN. Definitions of bloodstream infection in the newborn. Pediatr Crit Care Med. 2005 May; 6(3)(Suppl.): S45-9. DOI: 10.1097/01. PCC.0000161946.73305.0A.

44. Russell JA. Management of Sepsis. N Engl J Med. 2006 Oct; 355:1699-713. DOI: 10.1056/NEJMra043632.

45. Bhat R, Rao A. The Performance Of Haematological Screening Parameters And CRP In Early Onset Neonatal Infections. Journal of Clinical and Diagnostic Research. 2010 Dec; (4):3331-36. 
46. Tripathi S, Malik GK. Neonatal Sepsis: past, present and future; a review article. Internet Journal of Medical Update. 2010 July; 5(2):45-54. DOI: 10.4314/ijmu.v5i2.56163.

47. Simonsen KA, Anderson-Berry AL, Delair SF, Davies HD. Early-Onset Neonatal Sepsis. Clinical Microbiology Reviews. 2014 Jan; 27(1):21-47. DOI: 10.1128/CMR.00031-13.

48. Chirico G, Loda C. Laboratory aid to the diagnosis and therapy of infection in the neonate. Pediatric Reports. 2011 Feb; 3(1):e1. DOI: 10.4081/pr.2011.e1.

49. Shah BA, Padbury JF. Neonatal sepsis. Virulence. 2014 Jan; 5(1):170-8. DOI: 10.4161/viru.26906.

50. Makkar M, Gupta C, Pathak R, Garg S, Mahajan NC. Performance Evaluation of Hematologic Scoring System in Early Diagnosis of Neonatal Sepsis. J Clin Neonatol. 2013 Jan; 2(1): 25-9. DOI: 10.4103/2249-4847.109243.

51. Boseila S, Seoud I, Samy G, El-Gamal H, Ibrahim TS, Ahmed A, et al. Serum Neopterin Level in Early Onset Neonatal Sepsis. Journal of American Science. 2011; 7(7):343-52. (ISSN: 1545-1003).

52. Chiesa C, Panero A, Osborn JF, Simonetti AF, Pacifico L. Diagnosis of Neonatal Sepsis: A Clinical and Laboratory Challenge. Clin Chem. 2004 Feb; 50(2):279-87. DOI: 10.1373/clinchem.2003.025171.

53. Connell TG, Rele M, Cowley D, Buttery JP, Curtis N. How reliable is a negative blood culture result? Volume of blood submitted for culture in routine practice in a children's hospital. Pediatrics. 2007 May; 119(5):8916. DOI: $10.1542 /$ peds.2006-0440.

54. Selimovic A, Skokic F, Bazardzanovic M, Selimovic $Z$. The predictive score for early-onset neonatal sepsis. Turk J Pediatr. 2010 Mar-Apr; 52(2):139-44.

55. Mishra UK, Jacobs SE, Doyle LW, Garland SM. Newer approaches to the diagnosis of early onset neonatal sepsis. Arch Dis Child Fetal Neonatal Ed. 2006 May; 91(3):F208-F212. DOI: 10.1136/adc.2004.064188.

56. Polin RA, the Committee on Fetus and Newborn. Management of Neonates With Suspected or Proven Early-Onset Bacterial Sepsis. Pediatrics. 2012 May; 129(5):1006-15. DOI: 10.1542/peds.2012-0541.

57. Schmutz N, Henry E, Jopling J, Christensen RD. Expected Ranges for Blood Neutrophil Concentrations of Neonates: The Manroe and Mouzinho Charts Revisited. J Perinatol. 2008 Apr; 28(4):275-81. DOI: 10.1038/sj.jp.7211916.
58. Mouzinho A, Rosenfeld CR, Sanchez PJ, Risser R. Revised reference ranges for circulating neutrophils in very-low-birth-weight neonates. Pediatrics. 1994 Jul; 94:76-82.

59. 20. Sankar J, Agarwal R, Deorari AK, Paul VK. Sepsis in the newborn. Indian J of Pediatrics. 2008 Mar; 75(3):261-6. DOI: 10.1007/s12098-008-0056-z.

60. Murphy K, Weiner J. Use of leukocyte counts in evaluation of early-onset neonatal sepsis. Pediatr Infect Dis J. 2012 Jan; 31(1):16-9. DOI: 10.1097/ INF.0b013e31822ffc17.

61. Newman TB, Puopolo KM, Soora W, David D, Escobar GJ. Interpreting Complete Blood Counts Soon After Birth in Newborns at Risk for Sepsis. Pediatrics. 2010 Nov; 126(5):903-9. DOI: 10.1542/ peds.2010-0935.

62. Celik IH, Demirel G, Aksoy HT, Erdeve O, Tuncer E, Biyikli Z, Dilmen U. Automated determination of neutrophil VCS parameters in diagnosis and treatment efficacy of neonatal sepsis. Pediatric Research. 2012 Jan; 71(1):121-5. DOI: 10.1038/pr.2011.16.

63. Buttarello M, Plebani M. Automated blood cell counts: state of the art. Am J Clin Pathol. 2008 Jul; 130(1):10416. DOI: $10.1309 /$ EK3C7CTDKNVPXVTN.

64. da Silva O, Ohlsson A, Kenyon C. Accuracy of leucocyte indices and $\mathrm{C}$-reactive protein for diagnosis of neonatal sepsis: A critical review. Pediatr Infect Dis J. 1995 May; 14(5):362-6. DOI: 10.1097/00006454199505000-00005.

65. Sonawane VB, Gaikwad SU, Kadam NN, Gavhane J. Comparative Study of Diagnostic Markers in Neonatal Sepsis. J Nepal Paediatr Soc. 2014 May-August; 34(2):111-4. DOI: 10.3126/jnps.v34i2.9788.

66. Misra RN, Jadhav SV, Ghosh P, Gandham N, Angadi K, Vyawahare C. Role of sepsis screen in the diagnosis ofneonatal sepsis. Med J DY Patil Univ. 2013; 6(3):2547. DOI: $10.4103 / 0975-2870.114649$.

67. Borna H, Borna S. Value of Laboratory tests and C-Reactive Protein in the detection of Neonatal Sepsis. Internet J Pediatr Neonatol. 2005; 5:60-68.

68. Manzoni P, Mostert M, Galletto P, Gastaldo L, Gallo E, Agriesti G, Farina D, et al. Is thrombocytopenia suggestive of organism specific response in neonatal sepsis? Pediatr Int. 2009 Apr; 51(2):206-10. DOI: 10.1111/j.1442-200X.2008.02689.x. 
69. Mahmoud FM, Darwish NM, Hassan RA, Abou Shady NM. Evaluation of CD64 detection on neutrophils and TLR-2 on monocytes by flowcytometry as markers for early diagnosis of neonatal sepsis. International Journal of Advanced Research. 2014; 2(7):1235-47.

70. Kennon C, Overturf G, Bessman S, Sierra E, Smith KJ, Brann B. Granulocyte colony-stimulating factor as a marker for bacterial infection in neonates. $\mathrm{J}$ Pediatr. 1996 Jun; 128(6):765-9. DOI: 10.1016/S00223476(96)70327-X.

71. Usha P, Reddy S, Uma P, Lakshmi AB. Clinical correlation of neonatal and maternal hematological parameters as predictors of neonatal sepsis. IJRRMS. 2015 Jan-Mar; 5(1):1-8. 